\title{
Post-Caesarean analgesia in Timor-Leste, a low-middle-income country
}

\section{Dear Editor,}

Timor-Leste is a small, low-middle-income country in Southeast Asia. It is reported to spend less on healthcare than any other country. ${ }^{1}$ Hospital Nacional Guido Valadares performs 6000 deliveries a year. Epidural analgesia, intrathecal opiates, transversus abdominis plane blocks, oral and intravenous opiates, and non-steroidal anti-inflammatory agents have all been mooted as techniques useful in improving post-caesarean analgesia. ${ }^{2,3}$ Most of these options are unavailable in Timor-Leste. Opiate use in theatre is minimal and opiates are unavailable on the ward. Epidural anaesthesia and patient controlled analgesia are unavailable. A locally formulated post-caesarean pain management protocol, taking into account available agents and expertise, encourages spinal anaesthesia with hyperbaric bupivacaine followed by $100 \mathrm{mg}$ intramuscular tramadol in the recovery area, followed by oral paracetamol $1 \mathrm{gm}$ qid., tramadol $50 \mathrm{mg}$ qid. and ibuprofen $400 \mathrm{mg}$ tds. in the wards. This study sought to determine the efficacy of this protocol in a low resource environment.

Seventy women were assessed, undergoing either elective or emergency caesarean section. Choice of anaesthesia was at the discretion of the anaesthetist. A verbally reported pain score (0-10) was recorded on arrival in the recovery area and on the first postoperative morning. As per local practice the obstetrics team prescribed and administered analgesics in the ward. The patient chart was reviewed to determine which analgesics had been prescribed and which had been administered. Patients were asked if they were satisfied with their analgesia. Results are reported as mean (standard deviation).

The mean age of the participants was 28.4 (5.1) years. Sixtysix underwent spinal anaesthesia, of whom 4 received $25 \mu \mathrm{g}$ of intrathecal fentanyl. Nine (14\%) of the spinal anaesthetics required supplementation with ketamine to prolong anaesthesia. Eighteen women received intraoperative tramadol. Four women underwent general anaesthesia, and received $100 \mu \mathrm{g}$ of intravenous fentanyl. Two received a further $5 \mathrm{mg}$ of intravenous morphine. Mean surgical time was 66.7 (17.0) minutes. Mean pain score on arrival in the recovery area was 1.5 (2.0). Pain scores post-spinal anaesthesia were 1.4 (2.0), and post general anaesthesia 2.5 (1.9). Forty-eight women (68.6\%) were given analgesia in recovery - 33 intramuscular tramadol as per protocol, 2 intramuscular morphine, 2 oral paracetamol and 1 intravenous fentanyl.

On the first morning after caesarean section, overall verbally reported pain scores were 3.2 (1.7). Nineteen women had been prescribed and had received protocol analgesics, namely oral paracetamol, ibuprofen and tramadol. These women had pain scores of 2.4 (1.4). Thirty women had been prescribed and had received oral paracetamol and tramadol. Their pain scores were 3.2 (1.5), which were not significantly different from the scores in those receiving the full analgesia protocol $(P=0.07)$. Seventeen women received only some of their regular charted analgesics. This group had a pain score of 3.7 (1.8). Four women received no analgesia at all. Their mean pain score was 6.0 (1.7). No patient received 'prn' analgesia. 74\% of women reported being satisfied with their analgesia. Anecdotal observation suggested that as the study progressed, adherence to protocol and the administration of analgesics improved.

The challenges of improving perioperative care for women undergoing caesarean section in low-middle-income countries have been highlighted previously.,5,6 Adequate analgesia is important in enabling mothers to care for their babies and promptly return to full function. Cultural expectations are known to shape responses to pain management and the perception of whether or not pain is seen as a clinical problem. ${ }^{7}$ Despite the above, it was anecdotally observed that the obstetrics team and nursing staff became more aware of pain management and appeared more likely to prescribe and administer appropriate analgesics as the study progressed. This suggests that the performance of such investigations may make an important contribution to improvements in patient care. If anaesthetists are able to extend their clinical reach to the review of patients on the ward postoperatively, there will likely be improvements in care. High levels of maternal and perinatal morbidity and mortality are reported to occur on the wards postoperatively, especially in the developing world. ${ }^{8}$ In this regard, follow-up by the anaesthetist is likely to have benefits extending beyond pain management.

The United States Joint Commission on Accreditation of Healthcare organizations suggests a goal of uniformly low pain scores of no more than 3 out of $10 .{ }^{9}$ The only subgroup to achieve this was the group that was prescribed and received protocol analgesics, namely paracetamol, tramadol and ibuprofen. Patients who received only some of their prescribed analgesics had progressively worse pain scores. Predictably, the 4 women who received no postoperative pain relief had the worst pain scores. While this latter circumstance would not be tolerated in high-income countries, it is not uncommon in low-and middleincome countries. The causes of failure to administer pain relief are varied. They include staff education and attitude, high case load, and inadequate supplies of medication.

Despite the lack of opiates and frequent incomplete administration of prescribed analgesics, $74 \%$ of women reported satisfaction with their analgesia. The expression of satisfaction or otherwise with pain relief has a complex cultural overlay. Previous patient experience and expectations significantly influence the 
response to pain. ${ }^{7}$ To complicate matters, interpreters provided further insight into the cultural complexities, commenting that some women were afraid of being compelled to stay in hospital, expressing their dissatisfaction, or appearing weak in front of family members. These issues raise the spectre of underreporting of pain. In addition, cultural complexities extend to the attending staff. Despite several patients having an obvious requirement for analgesia, no patient received 'prn' analgesia. Some of the related issues involved nursing training and attitudes. Nurses frequently felt that "the patient has already had analgesia", and therefore the assumption was that they did not require further medication. The local custom of fasting patients for 6 hours postoperatively, also often prevented women from receiving timely oral analgesics.

This study suggests that provision of acceptable and adequate post-caesarean analgesia without intravenous or intramuscular opiates is possible in low resource environments. Adherence to simple locally formulated oral analgesia protocols improves post-caesarean section pain relief. In low- and middle-income countries, active involvement by anaesthesia staff in the ward may improve compliance with protocols, and raises staff awareness of the treatment of pain as an important therapeutic goal in the treatment of women post-caesarean section.

\section{Acknowledgements}

The author wishes to acknowledge and thank the anaesthetic and maternity ward staff of Hospital Nacional Guido Valadares for their assistance in the completion of this study.

\section{RF Grace}

Hospital Nacional Guido Valadares, Dili, Timor-Leste

Corresponding author, email: robertfgrace@gmail.com

\section{References}

1. Rensch S. Timor-Leste 'spends less than any other country on healthcare'. News and comment on global public financial management. Available from: https:// www.publicfinanceinternational.org/news/2018/03/timor-leste-spends-lessany-other-country-healthcare. Accessed Oct 2018.

2. Schewe JC, Komusin A, Zinserling J, Nadstawek J, Hoeft A, Hering R. Effects of spinal anaesthesia versus epidural anaesthesia for caesarean section on post-operative analgesic consumption and postoperative pain. Eur J Anesthesiol. 2009;26(1):52-9.

3. Paech MJ, McDonnell NJ, Sinha A, Baber C, Nathan EA. A randomized controlled trial of parecoxib, celecoxib and paracetamol as adjuncts to patient controlled epidural analgesia after caesarean delivery. Anaesth Intensive Care. 2014;42(1):15-22.

4. Lonnée HA, Madzimbamuto F, Erlandsen ORM, et al. Anesthesia for Cesarean Delivery: A cross-sectional survey of provincial, district, and Mission hospitals in Zimbawe. Anesth Analg. 2018;126(6):2056-64.

5. Vasco M, Pandya S,Van Dyk D, Bishop DG, Dyer RA. Maternal critical care in resource-limited settings. Narrative Review. Int J Obstet Anesth. 2019;37:86-95.

6. Yuill G, Amroyab A, Millar S, Vardapetyan E, Habib AS, Owen MD. Establishing Obstetric Anesthesiology Practice Guidelines in the Republic of Armenia: A global health collaboration. Anesthesiology. 2017;127(2):220-6.

7. Peacock S, Patel S. Cultural influences on Pain. Rev Pain. 2008;1(2):6-9.

8. Fenton PM, Whitty CJ, Reynolds F. Caesarean section in Malawi: prospective study of early maternal and perinatal mortality. BMJ. 2003;327:587.

9. The Joint Commission. Available from: https://www.jointcommission.org. Accessed Nov 2018. 